\title{
HIGH-FREQUENCY RADIATION AND TRITIUM CHANNEL
}

\author{
D.D. AFONICHEV \\ Institute for Metals Superplasticity Problems, RAS, 39 Khalturina, Ufa, 450001, Russia \\ E-mail: afon@imsp.da.ru
}

\begin{abstract}
In the process of deformation at a temperature $T=710^{\circ} \mathrm{C}$ of titanium alloy samples preliminarily saturated by deuterium a radiation, which is not a neutron flow was detected. Electromagnetic radiation in the range of radio frequencies was detected in titanium alloy samples in the process of their saturation by deuterium. The probable mechanism of its occurrence may be a retardation of charged particles in the metallic matrix.
\end{abstract}

\section{Introduction}

Our work consists of two parts. In the first stage, we carried out investigations of the influence of the deformation of titanium alloy samples saturated with deuterium on the occurrence of cold nuclear fusion. ${ }^{1}$ The basic results of these investigations were obtained in the years 1990-1992.

In the beginning of this work, we started with the assumption that the structure of metals influences the processes proceeding in a solid phase. Obviously, the intrinsic properties of a metallic matrix are responsible not only for the ability of a material to accumulate deuterons but also for the possibility of deuterons to move and interact. That is why the processes occurring during the cold fusion should be sensitive to the structure of the metals and alloys used.

As is known, plastic deformation changes the character of the distribution, and increases the density, of crystal lattice defects - dislocations, vacancies, stacking faults, and low and high angle grain boundaries. ${ }^{2}$ It is also well $\mathrm{known}^{3}$ that hydrogen isotopes, being interstitial atoms, interact with crystal lattice defects creating atmospheres of impurity atoms. One can expect that during plastic deformation the migration of these defects should influence the rate of the processes occurring during the fusion of deuteron nucleus.

\section{Neutron measurements}

In the initial stage of our investigations, we detected neutrons using a field monitor (RUP-1) with a fast-neutron counter. The radiation detected in this case correlated in time with processes occurring during the saturation by deuterium, or during the deformation of deuterium saturated samples. The titanium alloy VT9 (6.5Al$3.3 \mathrm{Mo}-1.5 \mathrm{Zr}-0.25 \mathrm{Si}$ ) was used for the investigations. For these studies, we use cylindrical samples with dimensions $10 \times 15 \mathrm{~mm}$, that were saturated by deuterium 


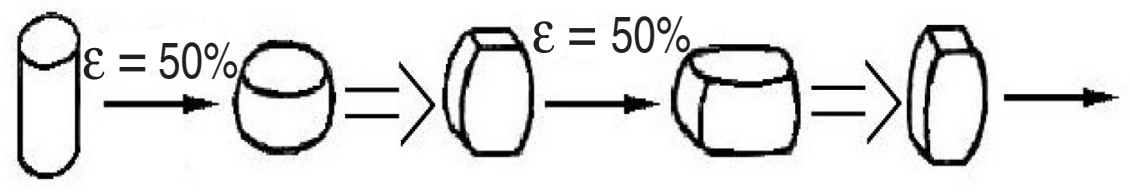

Figure 1. Scheme of deformation of samples by successive three-step compression.

from a gas phase up to concentration 2-20 at.\%. Deformation of the samples was carried out by compression uusing the scheme showed in Figure 1.

The temperature of deformation was selected on the basis of differential thermal analysis (DTA) data (Fig. 2). At the temperature $T \approx 650^{\circ} \mathrm{C}$, the weight of a sample starts to reduce, and a bend is observed on the DTA curve that testifies an intense escape of deuterium from a sample in the argon atmosphere. In the process of deformation of titanium alloy samples (at a temperature $T=710^{\circ} \mathrm{C}$ ), preliminarily saturated by deuterium, radiation, which is not a neutron flux, was detected. Figure 3 shows the time dependence of the load during deformation of the samples. Figure 4 presents the $(x-y)$ coordinate record of the emission detected by the field-effect monitor RUP-1. The break of deformation, or the increase in the strain rate by 10 times and more, resulted in a disappearance of the emission. In Fig. 3, the letters $\mathrm{A}$ and $\mathrm{B}$ indicate the time moments at which the traverse movement was stopped, and the same symbols indicate the periods of the absence of deformation in Fig. 4.

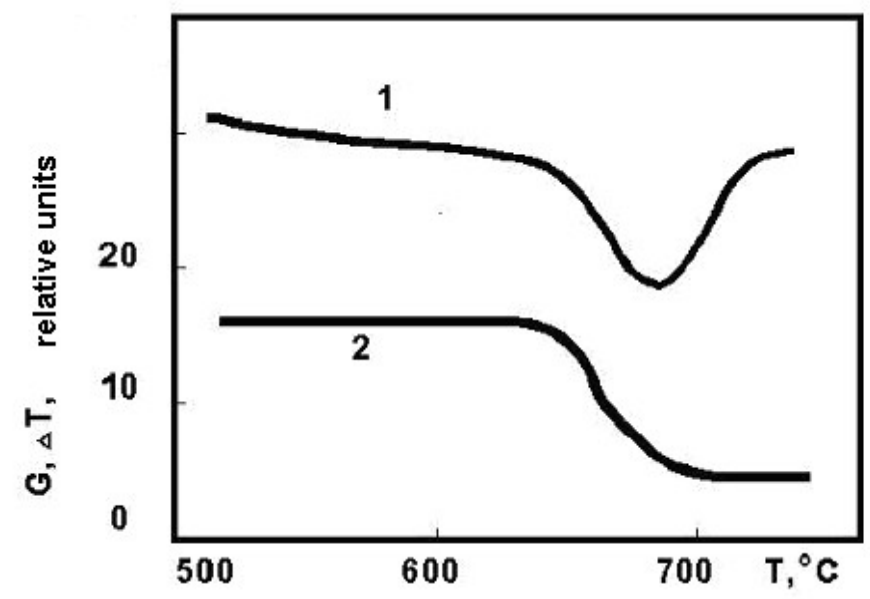

Figure 2. Temperature dependence of (1) differential thermal analysis and (2) weight of VT9 alloy sample, $C_{\mathrm{D}}=0,26$ at. $\%$. 


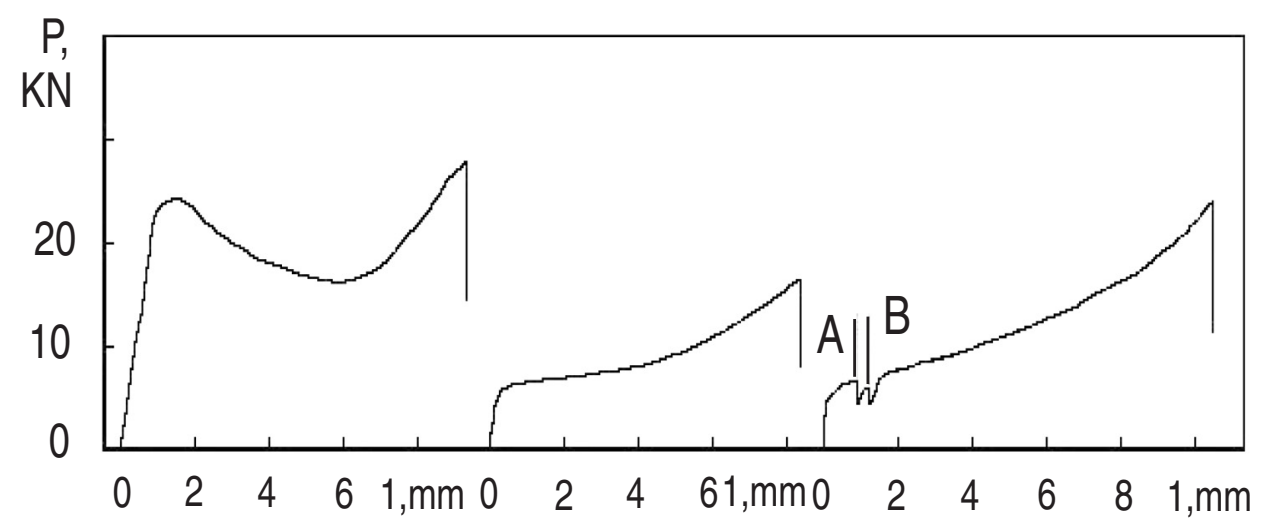

Figure 3. Load $(P)$ vs. elongation $(l)$ curves for successive three-step compression of VT9 alloy sample, $T=710^{\circ} \mathrm{C}$, concentration of deuterium $C_{\mathrm{D}}=12,3$ at. $\%, V=0,264 \mathrm{~mm} / \mathrm{min}$, A and $\mathrm{B}$ - points of deformation stop.

Two batches of VT9 alloy samples were studied. The first batch of samples have a structure with the size of primary $\beta$ grains $500 \pm 100 \mu \mathrm{m}, \alpha$ plate thickness of $6 \pm 1 \mu \mathrm{m}$, and the amount of $\beta$ phase about $20 \%$. Deuterium saturation of these samples did not lead to any significant changes in the structure, except for the growth of the amount of $\beta$ phase up to $50 \%$. The microstructure of the second batch of samples was characterized by the absence of $\alpha$ plates at matrix $\beta$ grain

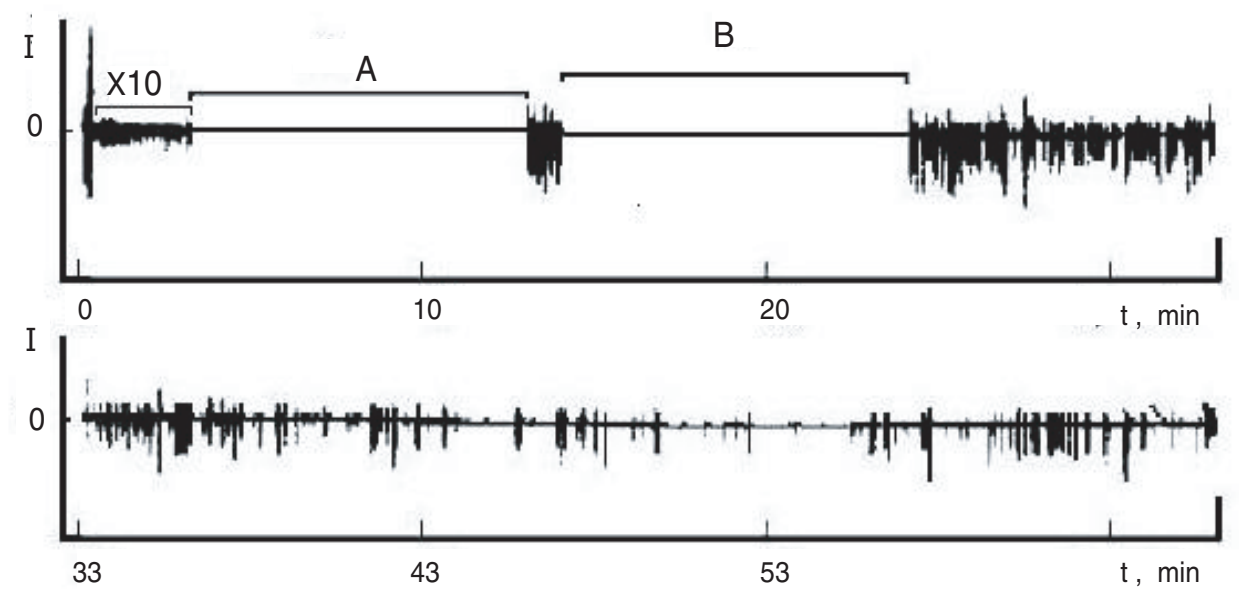

Figure 4. An experimental curve of the signal from a universal monitor RUP-1 with a neutron detector during the deformation of sample No. 2 in the third stage of deformation. $T=710^{\circ} \mathrm{C}$; $C_{\mathrm{D}}=12,3$ at. $\% ; V=0,264 \mathrm{~mm} / \mathrm{min}, \mathrm{A}$ and B-points of deformation stop. 
boundaries. Its $\alpha$ plates were bent and their thickness did not exceed $1 \pm 0.1 \mu \mathrm{m}$.

Three samples from the first batch were deformed at the temperate $T=710^{\circ} \mathrm{C}$, and radiation was registered from two of them. The concentration of deuterium in these samples was $C_{D}=11-12$ at.\%, while in the third sample it was considerably lower $-C_{\mathrm{D}}=8 \mathrm{at} . \%$. The samples of the second batch possessed more fine structure, and the radiation in these samples was detected only after their structure became similar to that of the first batch samples.

\section{Tritium measurements}

To confirm the occurrence of cold nuclear fusion, the measurement of tritium concentration was performed by the scintillation method via layerwise grinding. It was found that in all samples in which emission was detected during deformation, the concentration of tritium in the surface layer was increased by 3-7 times as compared to the background value. ${ }^{1}$ The energy spectrum of these samples corresponded to tritium.

We did not publish the results of this work in 1992 because the field monitor RUP-1 with a fast-neutron counter is not a reliable device to be used for scientific purposes. We have procured the radiometric device SRPS-2 having fast-neutron counters with a lamp SI-18H filled by ${ }^{3} \mathrm{He}$. Using this new device, we deformed about 50 samples but no appreciable neutron fluxes were registered.

After that, we conducted a joint experiment with a group of scientists from Ekaterinburg, on neutron detection in the process of saturation of our samples by deuterium. Whereas MKS-01 (a device similar to RUP-1) registered neutrons, the device SRPS-2 (the sensitivity of which is 100 times higher than that of MKS-01) did not register. Having performed this experiment we assumed that we registered electromagnetic radiation with a spectrum in the radio frequency regime. The tritium concentration was increased by $50 \%$ as compared to the background level evenly throughout the volume of this sample. The uniform distribution of tritium within the sample interior after its saturation by deuterium does not contradict the previous conclusion about the nonuniformity of the distribution, since a sufficiently high temperature applied during the present experiment leads to a convectiondiffusion leveling of the deuterium concentration.

\section{RF measurements}

In the second stage, we attempted the measurement of the (RF) radiation that we assumed was present during experiments in the first stage. In these experiments we attempted to measure radio-frequency radiation only during saturation of samples by deuterium, using various detection systems. Neutron detection with the radiometric device SRPS-2 was conducted during all our subsequent experiments.

Electromagnetic emission in the range of radio frequencies was detected by using different measuring devices: 
(1) A magnetic antenna with one turn in a symmetric copper ring with a notch. A signal from the antenna was transmitted to a wide-band amplifier UZ-29, then to an amplifier with a gain factor of $K=10^{4}$ (10 in a fourth degree), and after that to a ratemeter.

(2) A field meter M20 of optoelectronics (USA) with a telescopic antenna, $10 \mathrm{~cm}$ in length.

(3) An electron scheme comprising an antenna, $10 \mathrm{~cm}$ in length, a pulse amplifier with a gain factor of $K=5 \times 10^{3}$ (five increased on 10 in the third degree), discriminator and former drawing in a short signal for its recording.

For visualization of the emission detected in all cases, the signal is transmitted to an $x-y$ coordinate plotter.

Electromagnetic emission in the range of radio frequencies was detected during the saturation of titanium alloy VT-9 samples by deuterium from the gas phase at the temperature $T=860 \pm 10^{\circ} \mathrm{C}$. Prior to the saturation the samples were subjected to a bend strain at the room temperature in a three-point mode until the onset of plastic deformation.

$\mathrm{RF}$ emission has been detected under the conditions of continuously increasing pressure of deuterium. The rate of deuterium pressure increase was

$$
\frac{d p}{d t} \approx 0.8 \mathrm{kPa} / \mathrm{s} .
$$

The signal appeared at a pressure of $p \approx 24 \mathrm{kPa}$ and continued for $2-3 \mathrm{~min}$. RF emission was detected by means of each scheme described above. However, while in the case of the third scheme, the plotter recorded separate temporal resolution signals analogous to the field monitors RUP-1 and MKS-01; in case of the first two schemes a wide enveloping maximum was recorded due to the inertia of measuring systems. The RF emission had a sporadic character; the same character was noted when detecting the neutron flows in the first years of cold nuclear fusion study.

\section{Probable mechanism}

The generation of tritium while neutron flows are absent suggests that cold nuclear fusion occurs through a tritium channel as has been assumed earlier. ${ }^{4}$ The nonuniform formation of tritium within the sample interior after deformation testifies that the process of cold nuclear fusion occurs only in a surface layer. The same conclusion was also made in the review of early papers. ${ }^{5}$ The presence of electromagnetic radiation also confirms this conclusion, since the radio waves can be radiated only from near surface layers of metallic materials.

$\mathrm{RF}$ emission was detected only during the saturation of titanium alloy samples by deuterium, while during the saturation by conventional hydrogen it was not detected. We presume that the appearance of tritium in the samples where RF emission was detected points to the occurrence of cold nuclear fusion. 
We suggest that the most probable mechanism of the occurrence of RF emission, which accompanies cold nuclear fusion, is the interaction of products of nuclear reaction

$$
{ }^{2} \mathrm{D}+{ }^{2} \mathrm{D} \rightarrow{ }^{1} \mathrm{p}^{+}+{ }^{3} \mathrm{~T}^{+}+\mathrm{E}(4.03 \mathrm{Mev}),
$$

tritium and proton, with the metallic matrix. The movement of charged particles in the periodic field of the crystal lattice can result in the occurrence of RF emission. Unfortunately, we did not find articles about measurements of radiation within the range of radio frequencies when charged particles pass through the metallic matrix. The registration of such radiation can become indirect proof of the cold nuclear fusion occurrence along the tritium channel.

\section{Discussion}

Some authors account for the presence of tritium by the fact that its small amount is present in deuterium or in the metal under study. The preliminary preparation of samples in our experiments excluded the presence of tritium in the metal before its saturation by deuterium. During preliminary vacuum annealing up to $T=1200^{\circ} \mathrm{C}$ for $1 \mathrm{~h}$, the sample is completely clean from all hydrogen isotopes.

During the second stage of our work, we were not engaged in measurements of tritium. Now, we are trying to carry out new experiments to produce a significant amount of tritium. Only after successful termination of this experiment will we plan to start the measurement of heat effects.

The assumptions that have been presented in this work are not of a revolutionary nature. For their confirmation it is no need to create new physics. Simply this physics differs from the physics of hot nuclear fusion. In the former case, the processes occur in plasma. Cold nuclear fusion occurs in hard metal, under temperatures below $1000^{\circ} \mathrm{C}$.

\section{CONCLUSIONS}

(1) During successive three-step compression of preliminarily deuterium saturated VT9 alloy samples, an emission has been detected in the third stage of deformation, which is not a flow of neutrons or gamma quanta.

(2) In the surface layer of the samples for which the emission was detected, the concentration of tritium exceeded the background level by 3-7 times.

(3) The occurrence of emission and excess tritium concentration are associated with a specific structure of the samples, and observed only in the coarse-grained grosslamellar structure.

(4) During the saturation of VT-9 alloy samples by deuterium, an electromagnetic emission has been detected in the range of radio frequencies. Evidently, it was a pulse emission in the range of radio frequencies that was detected as neutrons during cold nuclear fusion by neutron monitors in early works. 


\section{ACKNOWLEDGMENT}

I would like to express the pleasure of an opportunity to see so many people, who trust in possibility of the cold fusion. I want also to express thanks to the organizers of the conferences for giving me possibility to be present at ICCF10.

\section{References}

1. D. D. Afonichev and M. A. Murzinova, Int. J. Hydrogen Energy, 28(9), 1005-1010 (2003).

2. O. A. Kaibyshev, Superplasticity of Alloys, Intermetallides and Ceramics (SpringerVerlag, Berlin, 1992, 317 pp).

3. Hydrogen in Metals, vol. 1, ed. G. Alefeld and I. Völkl (Springer, Berlin, Heidelberg, NY, 1978).

4. BARC Stadies in Cold Fusion, eds. P. K. Iengar and M. Srinivasan (Bhabha Atomic Research Center, BARC 1500, Trombay, Bombay, India, November 1989).

5. V. A. Tsarev, Sov. Phys. Usp., 35, 842 (1992). 